\title{
Coagulase-Negative Staphylococcus
}

National Cancer Institute

\section{Source}

National Cancer Institute. Coagulase-Negative Staphylococcus. NCI Thesaurus. Code C62584.

A subtype of Staphylococcus characterized by an inability to produce free plasma coagulase. 